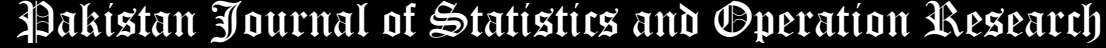

\section{A New Four Parameter Extended Exponential Distribution with Statistical Properties and Applications}

\author{
Amal Soliman Hassan ${ }^{1}$, Rokaya Elmorsy Mohamed ${ }^{2}$, Omid Kharazmi ${ }^{3}$, Heba \\ Fathy $\mathrm{Nagy}^{4^{*}}$
}

* Corresponding Author

1. Faculty of Graduate Studies for Statistical Research, Department of Mathematical Statistics, Cairo University, Egypt, amal52_soliman@cu.edu.eg

2. Sadat Academy for Management Sciences, Department of Mathematics, Statistics and Insurance, Cairo, Egypt, rokayaelmorsy@gmail.com

3. Faculty of Sciences, Department of Statistics, Vali-e-Asr University of Rafsanjan, Rafsanjan, Iran, omid.kharazmi@vru.ac.ir

4. Faculty of Graduate Studies for Statistical Research, Department of Mathematical Statistics, Cairo University, Egypt, heba_nagy_84@cu.edu.eg

\begin{abstract}
In this work, we introduce a novel generalization of the extended exponential distribution with four parameters through the Kumaraswamy family. The proposed model is referred to as the Kumaraswamy extended exponential (KwEE). The significance of the suggested distribution from its flexibility in applications and data modeling. As specific sub-models, it includes the exponential, Kumaraswamy exponential, Kumaraswamy Lindley, Lindley, extended exponential, exponentiated Lindley, gamma and generalized exponential distributions. The representation of the density function, quantile function, ordinary and incomplete moments, generating function, and reliability of the KwEE distribution are all derived. The maximum likelihood approach is used to estimate model parameters. A simulation study for maximum likelihood estimates was used to investigate the behaviour of the model parameters. A numerical analysis is performed for various sample sizes and parameter values to analyze the behaviour of estimates using accuracy measures. According to a simulated investigation, the KwEE's maximum likelihood estimates perform well with increased sample size. We provide two real-world examples utilizing applied research to demonstrate that the new model is more effective.
\end{abstract}

Key Words: Extended exponential distribution; Moments, Quantile; Maximum likelihood technique.

\section{Mathematical Subject Classification: 62F10}

\section{Introduction}

The exponential (E) distribution is the commonly used distribution for data analysis and is used in a variety of industries. However, in many practical cases, the exponential distribution can only be used with a constant hazard rate. Modified extensions of the exponential distribution have been created in contemporary statistical literature to overcome such challenges. For instance, exponentiated E (Gupta and Kundu (1999; 2001)), beta generalized E (Barreto-Souza et al. (2010)), gamma exponentiated E (Ristić and Balakrishnan (2012)), transmuted exponentiated E (Merovci (2013), exponentiated generalized extended E (de Andrade et al. (2016)), modified E (Rasekhi et al. (2017), odd exponentiated half-logistic E (Afify et al. (2018), Kumaraswamy extension E (Elbatal et al., 2018), alpha power extended E (Hassan et al. (2018)), Marshall-Olkin logistic-exponential alpha power E (Nassar et al. (2019)), extended odd Weibull E (Afify and Mohamed (2020)) distributions among others. 
Our interest here is in the extended exponential (EE) distribution prepared by Gómez et al. (2014), based on the extension of Nadarajah and Haghighi (2011), with the following cumulative distribution function (cdf) and probability density function (pdf)

$$
G(x ; \phi, \beta)=\frac{\phi+\beta-(\phi+\beta+\phi \beta x) e^{-\phi x}}{\phi+\beta}, x, \phi, \beta>0,
$$

and,

$$
g(x ; \phi, \beta)=\frac{\phi^{2}(1+\beta x) e^{-\phi x}}{\phi+\beta}, x, \phi, \beta>0 .
$$

The density (2) is a special case of the generalized Lindley (GL) distribution with scale parameters $\phi$ and $\beta$ (see Zakerzadeh and Dolati (2009)), where the authors do not address this particular case in their research. They proved that $\mathrm{EE}$ is a mixture of exponential and gamma distributions.

Many academics believe that extending and generalizing probability distributions can help them model a wider range of data in a variety of domains. One of the approaches that allows more flexible models is the use of generated families of probability distributions. The Kumaraswamy-G (Kw-G) family, with two parameters provided by Cordeiro and de Castro (2011) belong to a well-known family. The pdf and cdf of the Kw-G are defined by

$$
f(x)=v \delta g(x)(G(x))^{v-1}\left(1-(G(x))^{v}\right)^{\delta-1}
$$

and,

$$
F(x)=1-\left(1-(G(x))^{v}\right)^{\delta},
$$

where $G(x)$ is the baseline cdf, $v, \delta>0$, are two additional shape parameters. Clearly, for $v=\delta=0$, we obtain the baseline distribution $G(x)$. Equation (3) has the advantage of being able to fit skewed data that cannot be successfully fitted by existing distributions. Furthermore, it provides for higher tail flexibility and may be widely employed in many areas of reliability and biology, which is typically noticed in data sets emerging from sectors such as medicine, engineering, and so on. The composite between $\mathrm{Kw}$ and other distributions has gotten a lot of interest from researchers in the last few decades, and it's been extensively examined, such as Kw Gumbel distribution (Cordeiro et al. (2012)), Kw inverse Weibull distribution (Shahbaz et al. (2012)), Kw generalized Rayleigh distribution (Gomes et al. (2014)), Kw exponentiated Burr XII distribution (Afify and Mead (2017)), Kw inverted Topp-Leone distribution (Hassan et al. (2021)), and Kw Pareto IV distribution (Tahir et al., 2021).

It's worth noting that the EE distribution's hazard rate functions are limited, so it can't be used to represent lifetime data from medical, engineering, or other areas. To overcome this weakness, we propose the Kumaraswamy EE (KwEE), a generalization of the EE distribution via the Kw-G family. Furthermore, the KwEE distribution contains several important distributions, as cited in Table 1 of this paper, and as such, the proposed model supports a wide variety of shapes in terms of its probability density function plots as well as hazard rate function plots. This flexible nature of the proposed distribution can be expected to have extensive utility in modelling data sets from various fields of scientific research and has motivated us to investigate many useful properties of the distribution. We have illustrated the merit of the KwEE distribution over other existing models in Section 6 by considering two types of real data sets with an increasing hazard rate function. Furthermore, Tables 6 and 7 reveal that the KwEE distribution fits both types of data substantially better, demonstrating its versatility in modelling these data sets from reliability studies.

The following is an example of how to organize this paper. Section 2 contains a model description of the KwEE distribution, while Section 3 contains some structural aspects of the KwEE distribution. Parameter estimates and a simulation examination are discussed in Sections 4 and 5. The applications are presented in Section 6, followed by summary and conclusions.

\section{Model Description}

A four-parameter KwEE probability distribution is defined here, as well as its sub-models. The functions for reliability and hazard rate are also specified.

The cdf of the KwEE distribution is determined by substituting $G(x)$ in (4) with the cdf of the EE distribution as

$$
F(x ; \mathrm{M})=1-\left\{1-\left[1-\frac{(\phi+\beta+\phi \beta x) e^{-\phi x}}{\phi+\beta}\right]^{v}\right\}^{\delta}, x, v, \delta, \phi, \beta>0,
$$


where $\mathrm{M}=(\nu, \delta, \phi, \beta)$, is the set of parameters. The pdf that corresponds to (5) is:

$$
f(x ; \mathrm{M})=\frac{v \delta \phi^{2}(1+\beta x) e^{-\phi x}}{\phi+\beta}\left[1-\frac{(\phi+\beta+\phi \beta x) e^{-\phi x}}{\phi+\beta}\right]^{\nu-1}\left\{1-\left[1-\frac{(\phi+\beta+\phi \beta x) e^{-\phi x}}{\phi+\beta}\right]^{\nu}\right\}^{\delta-1} .
$$

Let $X \sim \operatorname{KwEE}(v, \delta, \phi, \beta)$ denotes a random variable with the pdf (6). The KwEE's special sub-models are listed in Table 1.

Table1: The KwEE distribution's sub-models

\begin{tabular}{|cccccc|}
\hline$v$ & $\delta$ & $\phi$ & $\beta$ & Reduced Model & Authors \\
\hline- & - & - & 0 & Kumaraswamy exponential (KwE) & Cordeiro and de Castro (2011) \\
- & - & - & 1 & Kumaraswamy Lindley (KwL) & Çakmakyapan and Kadilar (2014) \\
- & 1 & - & 0 & Generalized exponential (GE) & Gupta and Kundu (1999) \\
- & 1 & - & 1 & Exponentiated Lindley (EL) & Nadarajah et al. $(2011)$ \\
0 & 0 & - & - & Extended Exponential & Gómez et al. $(2014)$ \\
1 & 1 & - & 0 & Exponential & \\
1 & 1 & - & 1 & Lindley (L) & \\
\hline
\end{tabular}

Figure 1 illustrates some KwEE density charts with various shapes for various parameter values. The density (6) allows for greater flexibility depending on the parameter values, it can be unimodal, symmetric, reversed J-shaped, or right skewed, as seen in Figure 1.

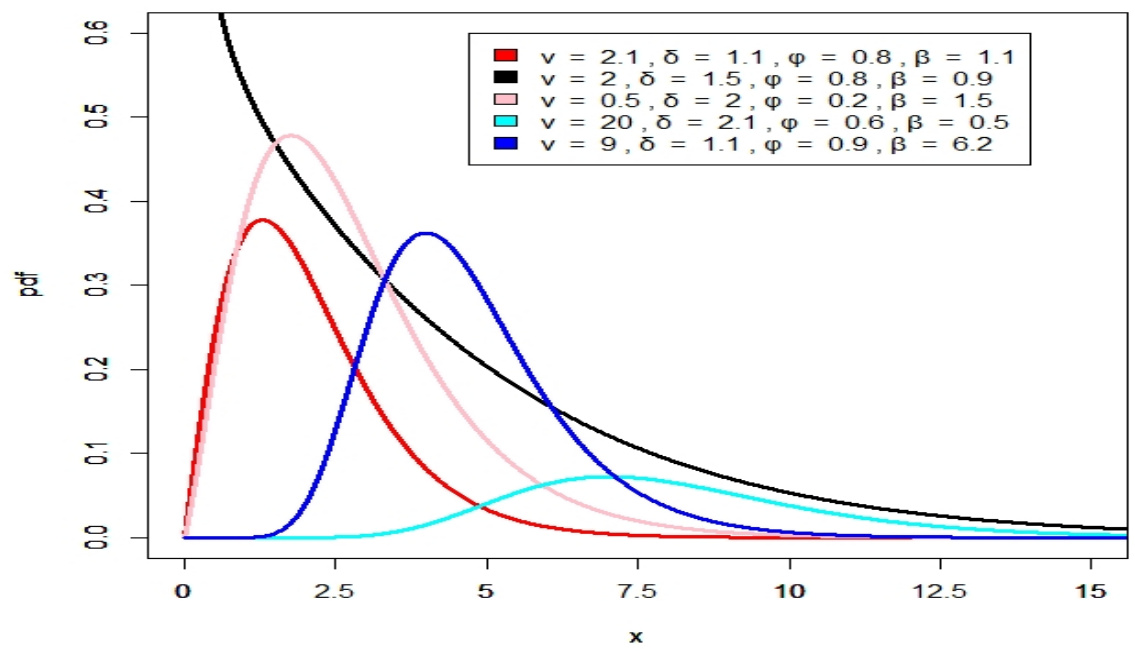

Figure 1: The KwEE density plots for specific values of parameters

The reliability function and hazard rate function (hrf) of $X$ are given, respectively, as follows:

$$
\bar{F}(x ; \mathrm{M})=\left\{1-\left[1-\frac{(\phi+\beta+\phi \beta x) e^{-\phi x}}{\phi+\beta}\right]^{v}\right\}^{\delta},
$$

and

$$
h(x ; \mathrm{M})=\frac{v \delta \phi^{2}(1+\beta x) e^{-\phi x}}{\phi+\beta}\left[1-\frac{(\phi+\beta+\phi \beta x) e^{-\phi x}}{\phi+\beta}\right]^{\nu-1}\left\{1-\left[1-\frac{(\phi+\beta+\phi \beta x) e^{-\phi x}}{\phi+\beta}\right]^{\nu}\right\}^{-1} .
$$

Figure 2 depicts some KwEE hrf charts with various shapes for various parameter values. Figure 2 shows that the hrf of the KwEE model can be constant-increasing $(v=1.5, \delta=2.5, \phi=0.2, \beta=1.5)$ or increasing $(\nu=0.5, \delta=2.5, \phi=3, \beta=0.5) \quad$ or $\quad$ decreasing $\quad(v=2.5, \delta=3, \phi=0.5, \beta=3), \quad$ or $\quad$ (reversed 
$(\nu=1.5, \delta=2.5, \phi=0.2, \beta=1.5)$. We can see from Figure 2 that the KwEE model may be used to model data using the indicated hrfs.

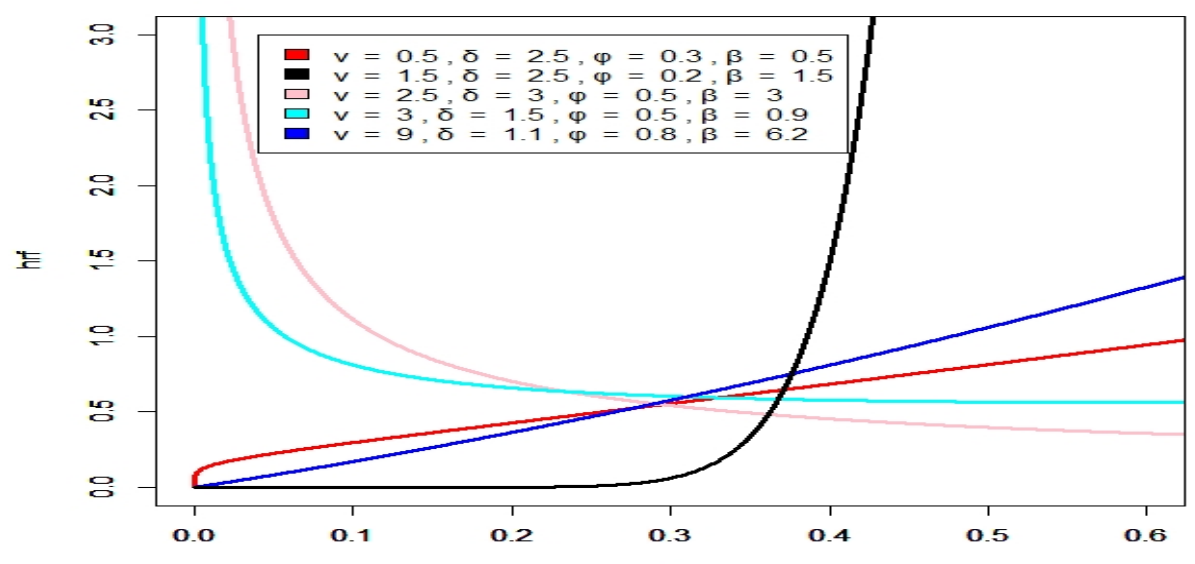

Figure 2: The KwEE hrf plots for specific values of parameters

\section{Major Properties}

We look at the pdf expansion, quantile function, moments, moment generating function, incomplete moments, and residual moments of the KwEE distribution.

\subsection{Expansion}

The binomial theorem is used to obtain a suitable expansion of the KwEE pdf, with binomial expansion given as

$$
(1-p)^{a-1}=\sum_{c=0}^{\infty}(-1)^{c}\left(\begin{array}{c}
a-1 \\
c
\end{array}\right) p^{c} .
$$

We get (7) by plugging it into pdf (6)

$$
f(x ; \mathrm{M})=\sum_{j=0}^{\infty}(-1)^{j}\left(\begin{array}{c}
\delta-1 \\
j
\end{array}\right) \frac{v \delta \phi^{2}(1+\beta x) e^{-\phi x}}{(\phi+\beta)^{v(j+1)}}\left[\phi+\beta-(\phi+\beta+\phi \beta x) e^{-\phi x}\right]^{v(j+1)-1} .
$$

Using binomial expansion once more

$$
f(x ; \mathrm{M})=\sum_{j, i=0}^{\infty} \sum_{k=0}^{i}\left(\begin{array}{c}
\delta-1 \\
j
\end{array}\right)\left(\begin{array}{c}
v(j+1)-1 \\
i
\end{array}\right)\left(\begin{array}{l}
i \\
k
\end{array}\right) \frac{(-1)^{i+j} \delta v \phi^{2}(1+\beta x) e^{-\phi(i+1) x}}{\phi+\beta}\left(\frac{\phi \beta x}{\phi+\beta}\right)^{k} .
$$

Hence, the previous pdf of KwEE distribution formatted as follows:

$$
f(x ; \mathrm{M})=\sum_{j, i=0}^{\infty} \sum_{k=0}^{i} W_{i, j} \frac{\delta v \phi^{2+k} \beta^{k}}{(\phi+\beta)^{k+1}}\left(x^{k}+\beta x^{k+1}\right) e^{-\phi(i+1) x},
$$

$$
\text { where, } \quad W_{i, j}=(-1)^{i+j}\left(\begin{array}{c}
\delta-1 \\
j
\end{array}\right)\left(\begin{array}{c}
v(j+1)-1 \\
i
\end{array}\right)\left(\begin{array}{l}
i \\
k
\end{array}\right) \text {. }
$$

\subsection{Quantile function}

It's crucial to know $X$ 's quantile function for a variety of applications. $X$ 's quantile function can be written as by inverting (5).

which yields;

$$
u=1-\left\{1-\left[1-\left(1+\frac{\phi \beta Q(u)}{\phi+\beta}\right) e^{-\phi Q(u)}\right]^{v}\right\}^{\delta}
$$




$$
-\left(\frac{\phi+\beta}{\beta}+\phi Q(u)\right) e^{-\left(\frac{\phi+\beta}{\beta}+\phi Q(u)\right)}=\frac{-(\phi+\beta)}{\beta}\left[1-\left\{1-[1-u]^{\frac{1}{\delta}}\right\}^{\frac{1}{v}}\right] e^{-\left(\frac{\phi+\beta}{\beta}\right)} .
$$

In above equation, we note that $-\left(\frac{\phi+\beta}{\beta}+\phi Q(u)\right)$ is Lambert $W$-function of the real argument.

Then, $W(x) e^{W(x)}=x$. function is defined by-Wambert LThe $\cdot \frac{-(\phi+\beta)}{\beta}\left[1-\left\{1-[1-u]^{\frac{1}{\delta}}\right\}^{\frac{1}{v}}\right] e^{-\left(\frac{\phi+\beta}{\beta}\right)}$

$$
-\left(\frac{\phi+\beta}{\beta}+\phi Q(u)\right)=W_{-1}\left[\frac{-(\phi+\beta)}{\beta}\left[1-\left\{1-[1-u]^{\frac{1}{\delta}}\right\}^{\frac{1}{v}}\right] e^{-\left(\frac{\phi+\beta}{\beta}\right)}\right] .
$$

Hence, we have the negative Lambert $W$-function of the real argument

$$
Q(u)=-\frac{1}{\beta}-\frac{1}{\phi}-\frac{1}{\phi} W_{-1}\left[\frac{-1}{\beta}(\phi+\beta)\left[1-\left\{1-[1-u]^{\frac{1}{\delta}}\right\}^{\frac{1}{v}}\right] e^{-\left(\frac{\phi+\beta}{\beta}\right)}\right],
$$

where $0<u<1$ and $W(\cdot)$ denotes the Lambert $W$-function. The Lambert $W$-function has been used to tackle a variety of issues in mathematics, physics, and engineering (Corless et al. (1996); Jodrá, 2010). According to de Andrade et al. (2016), the power series for the W-function holds as follows when utilizing the Lagrange inversion theorem.

$$
W(z)=\sum_{k=1}^{\infty} \frac{(-k)^{k-1} z^{k}}{k !}
$$

Applying (11) in (10), we have

$$
Q(u)=-\frac{1}{\beta}-\frac{1}{\phi}-\frac{1}{\phi} \sum_{k=1}^{\infty} \frac{(-1)^{k}(-k)^{k-1}}{k !} \frac{(\phi+\beta)^{k}}{\beta^{k}}\left[1-\left\{1-[1-u]^{\frac{1}{\delta}}\right\}^{\frac{1}{v}}\right]^{k} e^{-k\left(\frac{\phi+\beta}{\beta}\right)} .
$$

\begin{tabular}{|c|c|c|c|c|c|c|c|c|c|c|c|c|c|}
\hline \multirow{2}{*}{$v$} & \multirow{2}{*}{$\delta$} & \multicolumn{2}{|c|}{$\phi=0.5$} & \multicolumn{2}{|c|}{$\beta=1.5$} & \multicolumn{2}{|c|}{$\phi=1.5$} & \multicolumn{2}{|c|}{$\beta=0.5$} & \multicolumn{2}{|c|}{$\phi=2.5$} & \multicolumn{2}{|c|}{$\beta=1$} \\
\hline & & $50 \%$ & $75 \%$ & $85 \%$ & $95 \%$ & $50 \%$ & $75 \%$ & $85 \%$ & $95 \%$ & $50 \%$ & $75 \%$ & $85 \%$ & $95 \%$ \\
\hline \multirow{4}{*}{0.5} & 0.5 & 2.511 & 4.689 & 6.525 & 10.325 & 0.499 & 1.247 & 1.816 & 3.029 & 1.583 & 3.328 & 4.561 & 7.104 \\
\hline & 1 & 0.844 & 2.116 & 3.073 & 5.094 & 0.176 & 0.499 & 0.767 & 1.371 & 0.687 & 1.583 & 2.236 & 3.601 \\
\hline & 1.5 & 0.459 & 1.283 & 1.889 & 3.259 & 0.090 & 0.275 & 0.438 & 0.821 & 0.397 & 0.985 & 1.426 & 2.363 \\
\hline & 2 & 0.303 & 0.844 & 1.303 & 2.325 & 0.055 & 0.176 & 0.286 & 0.556 & 0.266 & 0.687 & 1.016 & 1.727 \\
\hline \multirow{4}{*}{1.5} & 0.5 & 3.991 & 6.623 & 8.433 & 12.169 & 1.037 & 1.847 & 2.421 & 3.628 & 2.858 & 4.627 & 5.839 & 8.336 \\
\hline & 1 & 2.476 & 3.991 & 4.998 & 7.025 & 0.598 & 1.342 & 1.037 & 1.342 & 1.830 & 2.858 & 3.536 & 4.896 \\
\hline & 1.5 & 1.891 & 3.010 & 3.741 & 5.189 & 0.438 & 0.749 & 0.963 & 1.400 & 1.427 & 2.194 & 2.689 & 3.665 \\
\hline & 2 & 1.565 & 2.476 & 3.065 & 4.216 & 0.353 & 0.598 & 0.765 & 1.105 & 1.201 & 1.830 & 2.231 & 3.010 \\
\hline
\end{tabular}

The median, third quartile and the percentage points at $85 \%$ and $95 \%$ are computed for some selected values of the parameters as seen in Table 2 .

Table 2: Percentage Points for $v, \delta, \phi$ and $\beta$

From Table 2 we notice that as the percentage level increases, the percentage values of the parameters also increase. As the value of $\delta$ increases, the percentage values decrease at all values of the parameters.

\subsection{Moments}

Moments are very valuable in statistical analysis, especially in applications. The formula for moments, incomplete moments of the KwEE distribution, is derived here. The $s^{\text {th }}$ moment for the KwEE distribution about zero is calculated as follows using pdf (9): 


$$
\mu_{s}^{\prime}=\sum_{j, i=0}^{\infty} \sum_{k=0}^{i} W_{i, j} \frac{v \delta \phi^{2+k} \beta^{k}}{(\phi+\beta)^{k+1}} \int_{0}^{\infty} x^{s}\left(x^{k}+\beta x^{k+1}\right) e^{-\phi(i+1) x} d x,
$$

which leads to

$$
\mu_{s}^{\prime}=\sum_{j, i=0}^{\infty} \sum_{k=0}^{i} W_{i, j} \frac{v \delta \phi^{2+k} \beta^{k}}{(\phi+\beta)^{k+1}}\left[\frac{\Gamma(s+k+1)}{(\phi(i+1))^{s+k+1}}+\frac{\beta \Gamma(s+k+2)}{(\phi(i+1))^{s+k+2}}\right], s=1,2, . .
$$

The first four moments about zero can be found by setting $s=1,2,3$ and 4 in (12). Also, the KwEE distribution's moment generating function can be found as follows:

$$
M_{x}(t)=E\left(\mathrm{e}^{t x}\right)=\sum_{s=0}^{\infty} \frac{t^{s}}{s !} \sum_{j, i=0}^{\infty} \sum_{k=0}^{i} W_{i, j} \frac{v \delta \phi^{2+k} \beta^{k}}{(\phi+\beta)^{k+1}}\left[\frac{\Gamma(s+k+1)}{(\phi(i+1))^{s+k+1}}+\frac{\beta \Gamma(s+k+2)}{(\phi(i+1))^{s+k+2}}\right] .
$$

The $s^{\text {th }}$ central moment $\left(\mu_{s}\right)$ of $X$ is given by

$$
\mu_{s}=E\left(X-\mu_{1}^{\prime}\right)^{s}=\sum_{i=0}^{s}(-1)^{i}\left(\begin{array}{l}
s \\
i
\end{array}\right)\left(\mu_{1}^{\prime}\right)^{i} \mu_{s-i}^{\prime}
$$

Furthermore, the $s^{\text {th }}$ incomplete moment, say $\varsigma_{s}(t)$ of the KwEE distribution can be calculated as follows

$$
\varsigma_{s}(t)=\sum_{j, i=0}^{\infty} \sum_{k=0}^{i} W_{i, j} \frac{v \delta \phi^{2+k} \beta^{k}}{(\phi+\beta)^{k+1}} \int_{0}^{s} x^{s}\left(x^{k}+\beta x^{k+1}\right) e^{-\phi(i+1) x} d x .
$$

Then,

\begin{tabular}{|c|c|c|c|c|c|c|}
\hline$\beta=1.5, \phi=2$ & $\mu_{1}^{\prime}$ & $\mu_{2}^{\prime}$ & $\mu_{3}^{\prime}$ & $\mu_{4}^{\prime}$ & Sk & $\mathrm{Ku}$ \\
\hline$v=0.5, \delta=1$ & 0.515 & 0.729 & 1.622 & 4.838 & 2.433 & 11.369 \\
\hline$v=0.5, \delta=1.5$ & 0.264 & 0.196 & 0.23 & 0.362 & 2.474 & 11.61 \\
\hline$v=0.5, \delta=2$ & 0.177 & 0.096 & 0.083 & 0.098 & 2.687 & 13.284 \\
\hline$v=0.5, \delta=2.5$ & 0.129 & 0.053 & 0.037 & 0.034 & 2.875 & 14.916 \\
\hline$v=1, \delta=1$ & 0.714 & 0.929 & 1.714 & 4.071 & 1.675 & 7.057 \\
\hline$v=1, \delta=1.5$ & 0.494 & 0.448 & 0.577 & 0.953 & 1.674 & 7.007 \\
\hline$v=1, \delta=2$ & 0.38 & 0.267 & 0.266 & 0.341 & 1.683 & 7.022 \\
\hline$v=1, \delta=2.5$ & 0.31 & 0.178 & 0.146 & 0.153 & 1.694 & 7.061 \\
\hline$v=1.5, \delta=1$ & 0.903 & 1.282 & 2.465 & 5.974 & 1.461 & 6.203 \\
\hline$v=1.5, \delta=1.5$ & 0.669 & 0.691 & 0.954 & 1.642 & 1.379 & 5.799 \\
\hline$v=1.5, \delta=2$ & 0.543 & 0.451 & 0.496 & 0.675 & 1.328 & 5.547 \\
\hline$v=1.5, \delta=2.5$ & 0.463 & 0.325 & 0.301 & 0.344 & 1.292 & 5.377 \\
\hline$v=2, \delta=1$ & 1.048 & 1.591 & 3.163 & 7.802 & 1.35 & 5.818 \\
\hline$v=2, \delta=1.5$ & 0.807 & 0.917 & 1.339 & 2.385 & 1.227 & 5.283 \\
\hline$v=2, \delta=2$ & 0.675 & 0.632 & 0.749 & 1.073 & 1.147 & 4.95 \\
\hline$v=2, \delta=2.5$ & 0.589 & 0.477 & 0.485 & 0.59 & 1.091 & 4.723 \\
\hline$v=2.5, \delta=1$ & 1.166 & 1.865 & 3.817 & 9.566 & 1.282 & 5.607 \\
\hline$v=2.5, \delta=1.5$ & 0.92 & 1.127 & 1.722 & 3.159 & 1.135 & 5.009 \\
\hline$v=2.5, \delta=2$ & 0.784 & 0.805 & 1.014 & 1.514 & 1.039 & 4.642 \\
\hline$v=2.5, \delta=2.5$ & 0.696 & 0.626 & 0.685 & 0.879 & 0.97 & 4.396 \\
\hline
\end{tabular}

$$
\varsigma_{s}(t)=\sum_{j, i=0}^{\infty} \sum_{k=0}^{i} W_{i, j} \frac{v \delta \phi^{2+k} \beta^{k}}{(\phi+\beta)^{k+1}}\left[\frac{\gamma(s+k+1,(\phi(i+1) t)}{(\phi(i+1))^{s+k+1}}+\beta \frac{\gamma(s+k+2,(\phi(i+1) t)}{(\phi(i+1))^{s+k+2}}\right] .
$$

where $\gamma(k, t)$ is the lower incomplete gamma function. Table 3 lists numerical values for the KwEE distribution's first four moments, skewness (Sk), and kurtosis $(\mathrm{Ku})$ for various parameter values.

Table 3: The KwEE distribution's moments measures 
We observe from Table 3 that with a constant value of $v$ and as the value of $\delta$ increases, all moments measures decerease. For the same value of $\delta$ and as the value of $v$ increases, all moments measures are increased. Based on the values of the $\mathrm{Sk}$ and $\mathrm{Ku}$ measurements, the KwEE distribution is right skewed and leptokurtic.

\subsection{Residual life and reversed failure rate function}

The $m^{\text {th }}$ moment of the residual life, $\Xi_{m}(t)=E\left[(X-t)^{m} \mid X>t\right], m=1,2, \ldots$ uniquely determines $F(x)$. The $m^{t h}$ moment of the residual life is defined by:

$$
\Xi_{m}(t)=\frac{1}{\bar{F}(t)} \int_{t}^{\infty}(x-t)^{m} f(x) d x .
$$

The $m^{\text {th }}$ moment of the residual life of the KwEE distribution is calculated using pdf (9)

$$
\begin{gathered}
\Xi_{m}(t)=\frac{1}{\bar{F}(t, \mathrm{M})} \sum_{j, i=0}^{\infty} \sum_{k=0}^{i} \sum_{r=0}^{m}(-1)^{m-r}\left(\begin{array}{c}
m \\
r
\end{array}\right) t^{m-r} W_{i, j} \frac{v \delta \phi^{2+k} \beta^{k}}{(\phi+\beta)^{k+1}}\left[\frac{\Gamma(r+k+1, \phi(i+1) t)}{(\phi(i+1))^{r+k+1}}\right. \\
\left.+\beta \frac{\Gamma(r+k+2, \phi(i+1) t)}{(\phi(i+1))^{r+k+2}}\right] .
\end{gathered}
$$

The mean residual life (MRL) function is another remarkable function with various applications in survival analysis in biological sciences, life insurance, maintenance, and so on. Setting $m=1$ in (13), will give the MRL of $X$.

The $m^{\text {th }}$ moment of the reversed residual life, say $\mathrm{K}_{m}(t)=E\left[(X-t)^{m} \mid X \leq t\right]$, for $t>0$ and $m=1,2, \ldots$ uniquely determines $F(x)$. Therefore, the $m^{\text {th }}$ moment of the reversed residual life of $X$ is given by

$$
\mathrm{K}_{m}(t)=\frac{1}{F(t)} \int_{0}^{t}(x-t)^{m} f(x) d x .
$$

The $m^{\text {th }}$ moment of the residual life of the KwEE distribution is calculated using pdf (9) as:

$$
\begin{aligned}
\mathrm{K}_{m}(t)=\frac{1}{F(t ; \mathrm{M})} \sum_{j, i=0}^{\infty} \sum_{k=0}^{i} \sum_{r=0}^{m}(-1)^{m-r}\left(\begin{array}{c}
m \\
r
\end{array}\right) t^{m-r} W_{i, j} \frac{v \delta \phi^{2+k} \beta^{k}}{(\phi+\beta)^{k+1}}\left[\frac{\gamma(r+k+1, \phi(i+1) t)}{(\phi(i+1))^{r+k+1}}\right. \\
\left.+\beta \frac{\gamma(r+k+2, \phi(i+1) t)}{(\phi(i+1))^{r+k+2}}\right] .
\end{aligned}
$$

The mean inactivity time (MIT) or mean waiting time, also known as the mean reversed residual life function, represents the amount of time that has passed since an item has failed under the assumption that it failed in $(0, t)$. By setting $m=1$ in the KwEE distribution, the MIT may be easily obtained from (14).

\section{Parameter Estimators}

The KwEE distribution parameters' maximum likelihood (ML) estimators are obtained. Let $X_{1}, \ldots, X_{n}$ represent observed values from the $\mathrm{KwEE}$ distribution with a set of parameters $\mathrm{M}=(v, \delta, \phi, \beta)^{T}$. The log-likelihood function for $\mathrm{M}$, may be stated as

$$
\begin{aligned}
\ln l= & n \ln v+n \ln \delta+2 n \ln \phi-n \ln (\phi+\beta)-\phi \sum_{i=1}^{n} x_{i}+\sum_{i=1}^{n} \ln \left(1+\beta x_{i}\right) \\
& +(v-1) \sum_{i=1}^{n} \ln \left(1-\left(1+D_{i}\right) e^{-\phi x_{i}}\right)+(\delta-1) \sum_{i=1}^{n} \ln \left[1-\left(1-\left(1+D_{i}\right) e^{-\phi x_{i}}\right)^{v}\right],
\end{aligned}
$$




$$
\begin{aligned}
& D_{i}=\frac{\phi \beta x_{i}}{\phi+\beta} \text {. The components of the score vector, } Z(\mathrm{M})=\left(\frac{\partial \ln l}{\partial v}, \frac{\partial \ln l}{\partial \delta}, \frac{\partial \ln l}{\partial \phi} \frac{\partial \ln l}{\partial \beta}\right)^{T} \text { are given by } \\
& Z_{v}=\frac{n}{v}+\sum_{i=1}^{n} \ln \left(1-\left(1+D_{i}\right) e^{-\phi x_{i}}\right)-(\delta-1) \sum_{i=1}^{n} \frac{\ln \left(1-\left(1+D_{i}\right) e^{-\phi x_{i}}\right)}{\left[\left(1-\left(1+D_{i}\right) e^{-\phi x_{i}}\right)^{-v}-1\right]} \\
& Z_{\delta}=\frac{n}{\delta}+\sum_{i=1}^{n} \ln \left[1-\left(1-\left(1+D_{i}\right) e^{-\phi x_{i}}\right)^{v}\right] \\
& Z_{\phi}=\frac{2 n}{\phi}-\frac{n}{\phi+\beta}-\sum_{i=1}^{n} x_{i}+(v-1) \sum_{i=1}^{n} \frac{\left(1+D_{i}\right) e^{-\phi x_{i}} x_{i}-e^{-\phi x_{i}} \partial D_{i} / \partial \phi}{1-\left(1+D_{i}\right) e^{-\phi x_{i}}} \\
& -(\delta-1) \sum_{i=1}^{n} \frac{v\left(1-\left(1+D_{i}\right) e^{-\phi x_{i}}\right)^{\nu-1}\left[\left(1+D_{i}\right) e^{-\phi x_{i}} x_{i}-e^{-\phi x_{i}} \partial D_{i} / \partial \phi\right]}{1-\left(1-\left(1+D_{i}\right) e^{-\phi x_{i}}\right)^{v}}, \\
& Z_{\beta}=\frac{-n}{\phi+\beta}+\sum_{i=1}^{n} \frac{x_{i}}{1+\beta x_{i}}-(v-1) \sum_{i=1}^{n} \frac{\left(\partial D_{i} / \partial \beta\right) e^{-\phi x_{i}}}{1-\left(1+D_{i}\right) e^{-\phi x_{i}}}+(\delta-1) \sum_{i=1}^{n} \frac{v\left(1-\left(1+D_{i}\right) e^{-\phi x_{i}}\right)^{v-1}\left(\partial D_{i} / \partial \beta\right) e^{-\phi x_{i}}}{1-\left(1-\left(1+D_{i}\right) e^{-\phi x_{i}}\right)^{v}}, \\
& \frac{\partial D_{i}}{\partial \beta}=\frac{\phi^{2} x_{i}}{(\phi+\beta)^{2}}, \frac{\partial D_{i}}{\partial \phi}=\frac{\beta^{2} x_{i}}{(\phi+\beta)^{2}} .
\end{aligned}
$$

The ML estimators, say $\hat{\mathrm{M}}=(\hat{v}, \hat{\delta}, \hat{\phi}, \hat{\beta})^{T}$, are obtained by setting the nonlinear system of equations $Z_{v}, Z_{\delta}, Z_{\phi}$ and $Z_{\beta}$ to zero and solving them simultaneously.

\section{Simulation Study}

We conduct a simulation analysis in this part to assess the performance of ML estimates (MLEs) in terms of absolute biases (ABs), standard errors (SEs), and mean squared errors (MSEs) for various sample sizes and parameter values. The simulation method is as follows

* From the KwEE distribution, generate 1000 random sample with sizes $n=10,20,30,50$, and 100 .

* Choose the following parameter values

Set $1 \equiv(\phi=0.3, \beta=0.2, v=0.8, \delta=0.5)$, Set $2 \equiv(\phi=0.5, \beta=0.5, v=0.6, \delta=0.8)$,

Set $3 \equiv(\phi=0.1, \beta=0.5, v=1.2, \delta=2)$ and $\operatorname{Set} 4 \equiv(\phi=1.2, \quad \beta=0.2, v=2, \delta=1.5)$

* For each model parameter and sample size, the MLEs of parameters are determined.

* The ABs, SEs, and MSEs of MLEs are then computed. The formula of ABs, SEs and MSEs are defined as follows

$$
\mathrm{ABs}=\left|\frac{1}{1000} \sum_{i=1}^{1000}\left(\hat{\theta}_{i}-\theta\right)\right|, \mathrm{MSEs}=\frac{1}{1000} \sum_{i=1}^{1000}\left(\hat{\vartheta}_{i}-\theta\right)^{2}, \mathrm{SEs}=\sqrt{\frac{1}{1000} \sum_{i=1}^{1000}\left(\hat{\vartheta}_{i}-\theta\right)^{2}},
$$

where, $\hat{\vartheta}$ is the estimates of the parameter $\vartheta$. From numerical outcomes in Tables 4 and 5, we notice the following

- The ABs and MSEs decrease with increases $n$ (see Tables 4, 5 and Figures 3, 4).

- As the value of $\phi$ gets larger, the $\mathrm{ABs}$ of $\hat{v}$ decrease. In almost estimates, the $\mathrm{ABs}$ of $\hat{\beta}$ increase as $\phi$ value increases.

- In almost all cases, the ABs and MSEs decrease as values of $v$ and $\delta$ increase for a fixed value of $\beta$ (see Tables 4 and 5). 


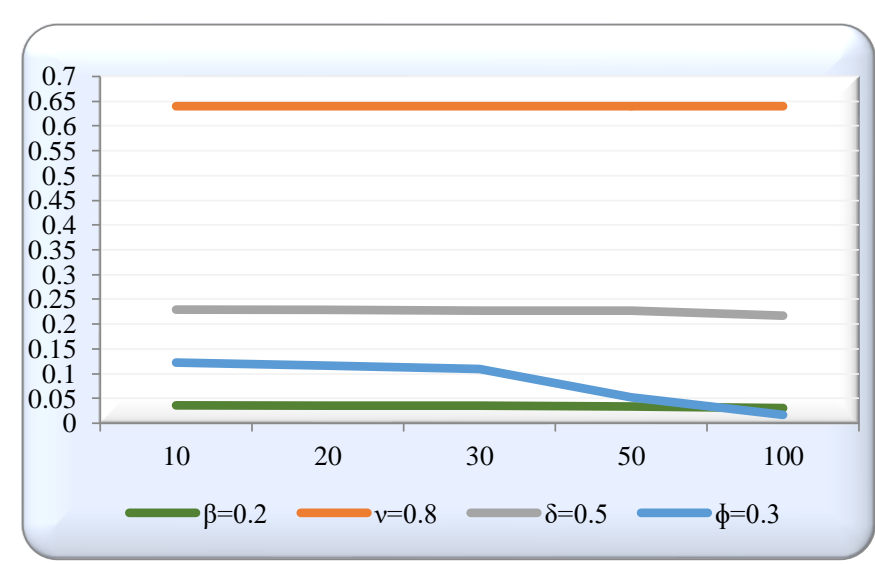

Figure 3: MSEs of MLEs for the Set 1

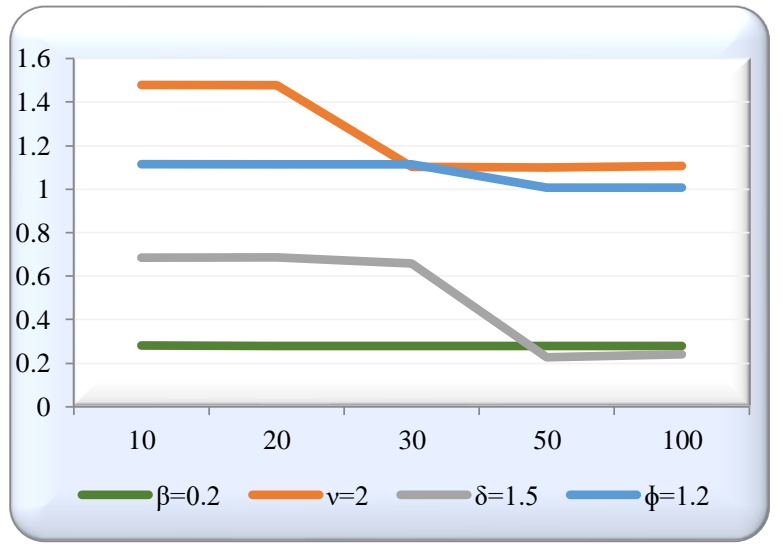

Figure 4: $\mathrm{ABs}$ of MLEs for the Set 4

Table 4: ABs, SEs and MSEs of the KwEE parameters

\begin{tabular}{|c|c|c|c|c|c|c|c|c|c|}
\hline \multirow[b]{2}{*}{$n$} & \multirow[b]{2}{*}{ Measure } & \multicolumn{4}{|c|}{ Set 1} & \multicolumn{4}{|c|}{ Set 2} \\
\hline & & $\hat{\phi}$ & $\hat{\beta}$ & $\hat{v}$ & $\hat{\delta}$ & $\hat{\phi}$ & $\hat{\beta}$ & $\hat{v}$ & $\hat{\delta}$ \\
\hline \multirow{4}{*}{10} & MSE & 0.1225 & 0.0361 & 0.6400 & 0.2294 & 0.2953 & 0.3221 & 0.2176 & 0.2089 \\
\hline & $\mathrm{AB}$ & 0.3500 & 0.1900 & 0.8000 & 0.4790 & 0.5432 & 0.5624 & 0.4984 & 0.6106 \\
\hline & SE & 0.0000 & 0.0000 & 0.0000 & 0.0000 & 0.0023 & 0.0021 & 0.0010 & 0.0045 \\
\hline & MSE & 0.1156 & 0.0357 & 0.6400 & 0.2285 & 0.2947 & 0.3087 & 0.0539 & 0.0514 \\
\hline \multirow[t]{2}{*}{20} & $\mathrm{AB}$ & 0.3400 & 0.1890 & 0.8000 & 0.4780 & 0.5429 & 0.5556 & 0.2318 & 0.2266 \\
\hline & SE & 0.0000 & 0.0000 & 0.0000 & 0.0000 & 0.0020 & 0.0020 & 0.0119 & 0.0089 \\
\hline \multirow{3}{*}{30} & MSE & 0.1089 & 0.0353 & 0.6400 & 0.2275 & 0.2947 & 0.3088 & 0.0537 & 0.0513 \\
\hline & $\mathrm{AB}$ & 0.3300 & 0.1880 & 0.8000 & 0.4770 & 0.5428 & 0.5557 & 0.2315 & 0.2263 \\
\hline & SE & 0.0000 & 0.0000 & 0.0000 & 0.0000 & 0.0004 & 0.0004 & 0.0004 & 0.0014 \\
\hline \multirow{3}{*}{50} & MSE & 0.0529 & 0.0342 & 0.6400 & 0.2266 & 0.2946 & 0.3088 & 0.0534 & 0.0511 \\
\hline & $\mathrm{AB}$ & 0.2300 & 0.1850 & 0.8000 & 0.4760 & 0.5428 & 0.5557 & 0.2312 & 0.2261 \\
\hline & SE & 0.0000 & 0.0000 & 0.0000 & 0.0000 & 0.0000 & 0.0000 & 0.0000 & 0.0011 \\
\hline \multirow{3}{*}{100} & MSE & 0.0169 & 0.0306 & 0.6400 & 0.2172 & 0.2945 & 0.3085 & 0.0533 & 0.0435 \\
\hline & $\mathrm{AB}$ & 0.1300 & 0.1750 & 0.8000 & 0.4660 & 0.5426 & 0.5554 & 0.2308 & 0.2086 \\
\hline & SE & 0.0000 & 0.0000 & 0.0000 & 0.0000 & 0.0000 & 0.0000 & 0.0000 & 0.0000 \\
\hline
\end{tabular}

Table 5: ABs, SEs and MSEs of the KwEE parameters

\begin{tabular}{|c|c|c|c|c|c|c|c|c|c|}
\hline \multirow[b]{2}{*}{$n$} & \multirow{2}{*}{ Measure } & \multicolumn{4}{|c|}{ Set 3} & \multicolumn{4}{|c|}{ Set 4} \\
\hline & & $\hat{\phi}$ & $\hat{\beta}$ & $\hat{v}$ & $\hat{\delta}$ & $\hat{\phi}$ & $\hat{\beta}$ & $\hat{v}$ & $\hat{\delta}$ \\
\hline \multirow{3}{*}{10} & MSE & 0.0042 & 0.2866 & 1.1521 & 2.4012 & 1.2426 & 0.0797 & 2.1949 & 0.4910 \\
\hline & SE & 0.0013 & 0.0013 & 0.0181 & 0.0153 & 0.0185 & 0.0169 & 0.0950 & 0.1509 \\
\hline & MSE & 0.0042 & 0.2853 & 1.1329 & 2.3587 & 1.2412 & 0.0781 & 2.1816 & 0.4725 \\
\hline 20 & $\mathrm{AB}$ & 0.0646 & 0.5341 & 1.0567 & 1.5357 & 1.1142 & 0.2795 & 1.4768 & 0.6865 \\
\hline \multirow{3}{*}{30} & MSE & 0.0042 & 0.2844 & 1.1101 & 2.3359 & 1.2207 & 0.0781 & 1.3083 & 0.4432 \\
\hline & $\mathrm{AB}$ & 0.0644 & 0.5333 & 1.0534 & 1.5281 & 1.1142 & 0.2795 & 1.1012 & 0.6572 \\
\hline & SE & 0.0021 & 0.0021 & 0.0203 & 0.0273 & 0.0015 & 0.0037 & 0.3092 & 0.5621 \\
\hline \multirow{3}{*}{50} & MSE & 0.0036 & 0.2842 & 1.0346 & 2.1055 & 1.0171 & 0.0781 & 1.2537 & 0.3750 \\
\hline & $\mathrm{AB}$ & 0.0598 & 0.5330 & 1.0170 & 1.4509 & 1.0079 & 0.2795 & 1.0994 & 0.2276 \\
\hline & $\mathrm{SE}$ & 0.0020 & 0.0021 & 0.0193 & 0.0240 & 0.0351 & 0.0037 & 0.2124 & 0.5685 \\
\hline
\end{tabular}




\section{Data Analysis}

Fitting the proposed distribution to two real data sets, we demonstrate the utility of the KwEE distribution. The $\mathrm{KwEE}$ distribution is compared to KwE, gamma, GE, one parameter L, GL, and EL models.

\section{(i) Data I}

The breaking stress of carbon fibers (in Gba) reported by Cordeiro and Lemonte (2011) is represented by this real data set. In the applications, the information about the hazard shape can help in selecting a particular model. For this aim, the total time test (TTT) plot (see Aarset (1987)) is an essential graphical technique to check if the data can be applied to a given distribution or not, this plot's is given by plotting

$$
G(r / n)=\left(\left(\sum_{i=1}^{r} x_{(i)}\right)+(n-r) x_{(r)}\right) /\left(\sum_{i=1}^{n} x_{(i)}\right), \quad r=1,2,3, \ldots n, \text { against } r / n, \text { and } x_{(i)},(i=1, \ldots, n) \text { are the order }
$$

statistics of the sample. The hrf is constant if the TTT plot is graphically displayed as a straight diagonal, but increasing (or decreasing) if the TTT plot is concave (or convex). If the TTT plot is initially convex and then concave, the hrf is U-shaped (bathtub); otherwise, the hrf is unimodal. The scaled-TTT plot in Figure 5 is concave. It shows that the hrf is increasing, proving the validity of our model.

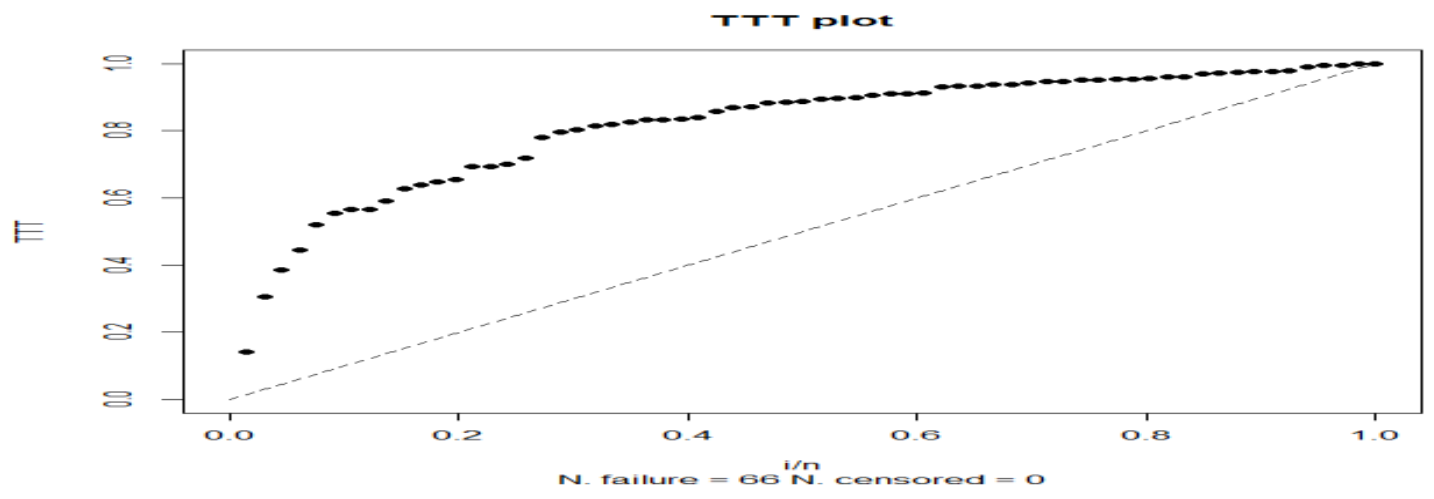

Figure 5: Scaled-TTT plot of the carbon data set

Table 6 shows the MLEs for the carbon data set, as well as the Akaike information criterion (AIC), Bayesian information criterion (BIC), Cramer-von Mises statistic ( $\left.\mathrm{W}^{*}\right)$, and Anderson-Darling statistic (A*). The better the fit, the lower the numerical criteria values. Table 6 shows that the KwEE model, out of all the models used, provides the best fit. In comparison to the other models, the KwEE model has the smallest AIC, BIC, A*, and W*.

Table 6: The MLEs and the proposed measures for carbon data

\begin{tabular}{|lllllll|}
\hline Model & \multicolumn{2}{c}{ MLEs } & AIC & BIC & \multicolumn{1}{c|}{$\boldsymbol{A}^{*}$} & $\boldsymbol{W}^{*}$ \\
\hline KwEE & $\hat{v}=3.016$ & $\begin{array}{l}\hat{\delta}=33.90 \\
\hat{\phi}=0.301\end{array}$ & $\mathbf{1 8 0 . 5 8 0}$ & $\mathbf{1 8 9 . 3 3 7}$ & $\mathbf{0 . 5 4 2}$ & $\mathbf{0 . 0 9 8}$ \\
\hline gamma & $\hat{\phi}=7.487$ & $\hat{\lambda}=2.713$ & 186.335 & 190.714 & 1.311 & 0.246 \\
\hline GE & $\hat{\phi}=9.201$ & $\hat{\lambda}=1.007$ & 194.745 & 199.124 & 2.094 & 0.373 \\
\hline KwE & $\hat{\phi}=10.424$ & $\hat{\beta}=1.235$ & 193.360 & 197.740 & 2.120 & 0.424 \\
\hline L & $\hat{\theta}=1.623$ & & 246.768 & 248.958 & 10.692 & 2.091 \\
\hline GL & $\hat{\theta}=2.783$ & $\hat{\phi}=6.904$ & 187.860 & 194.429 & 1.286 & 0.242 \\
& $\hat{\beta}=9.725$ & & & & & \\
\hline EL & $\hat{\theta}=1.246$ & $\hat{\phi}=7.042$ & 191.594 & 195.973 & 1.286 & 0.328 \\
\hline
\end{tabular}


A probability plot (P-P), often known as a probability plot, is a simple tool for detecting if a data set fits a hypothesized distribution. The data are plotted against a theoretical distribution in such a way that if the plot is a straight line, it's safe to believe the statistical sample comes from the stated distribution. Departures from this line denote deviations from the underlying distribution. Furthermore, the quantile-quantile (Q-Q) plot is a graphical tool for detecting whether two data sets are from the same population. A Q-Q plot is a plot of the quantiles of the first data set against the quantiles of the second data set.

As a result, we may conclude that the KwEE model is the best. Figure 6 shows the fitted pdfs, cdfs, P-P and Q-Q plots for all competitive distributions of the first data set. With a focus on the KwEE model, the fitted pdfs, cdfs, PP and Q-Q plots for the considered data is displayed in Figure 7. They all indicate how well the KwEE model fits the data, indicating its potential for use by practitioners in data analysis. Table 6's conclusions are also validated by these graphs.

Histogram and theoretical densities

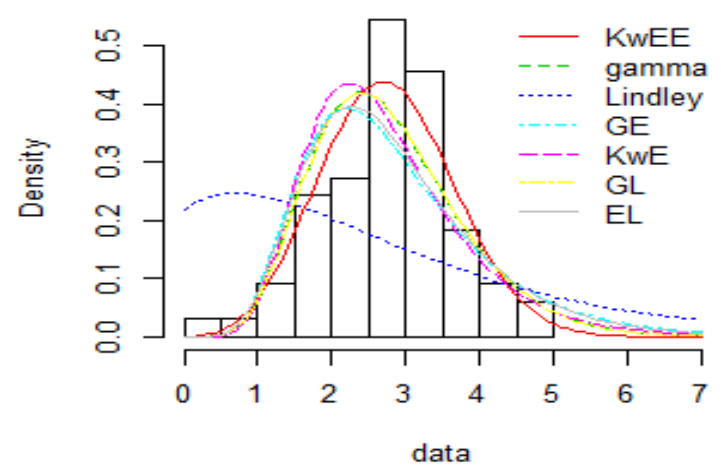

Empirical and theoretical CDFs

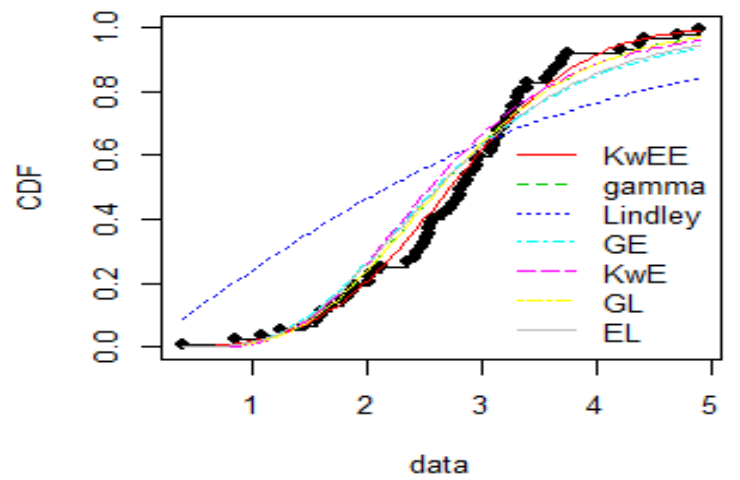

Q-Q plot

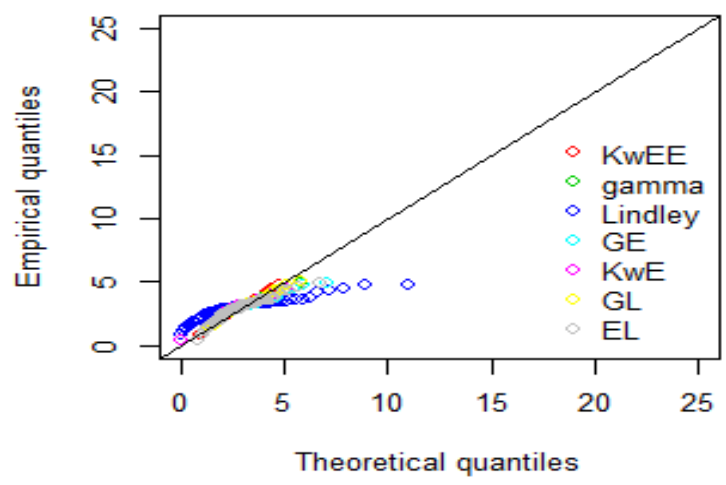

P-P plot

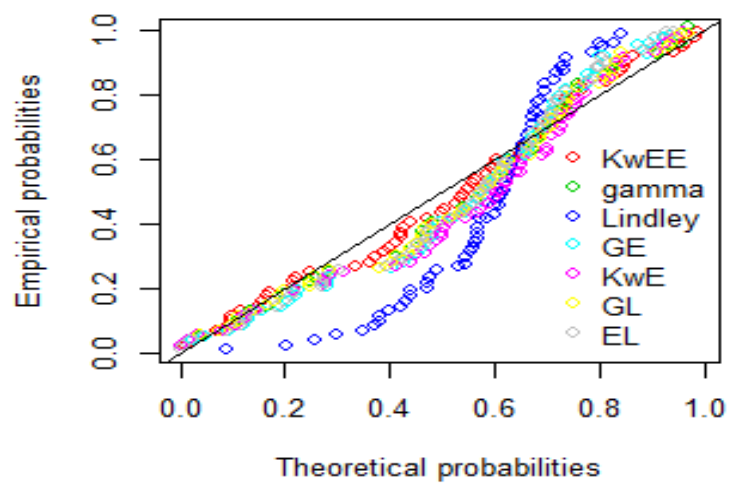

Figure 6:. Histogram and fitted density plots, the plots of empirical and fitted cdfs, P-P plots and Q-Q plots for the carbon data set 
Histogram and theoretical densities

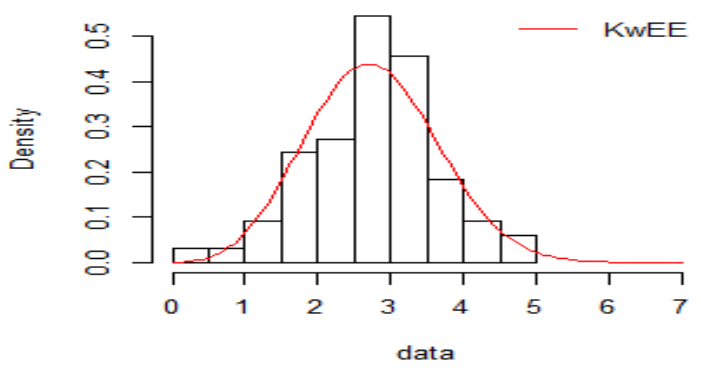

Empirical and theoretical CDFs

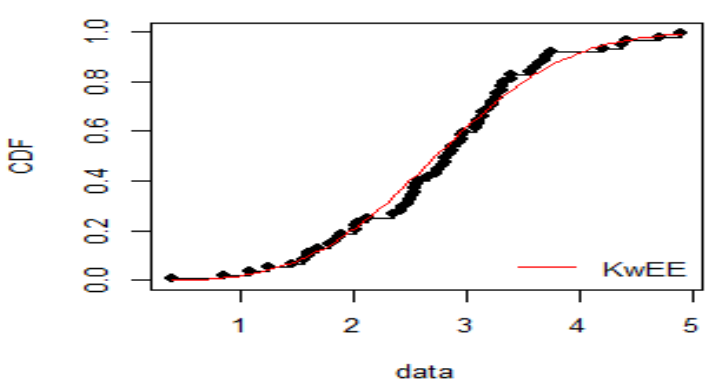

Q-Q plot

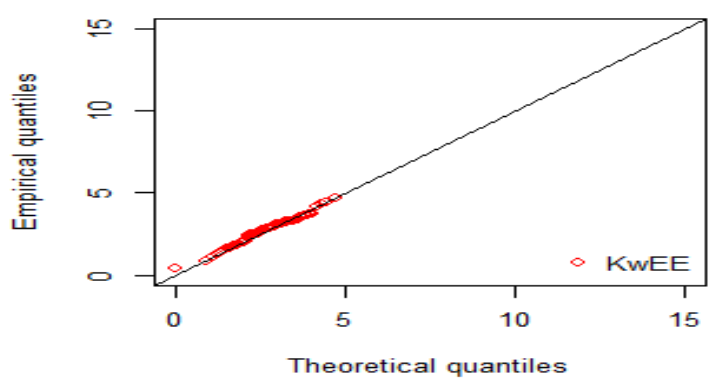

P-P plot

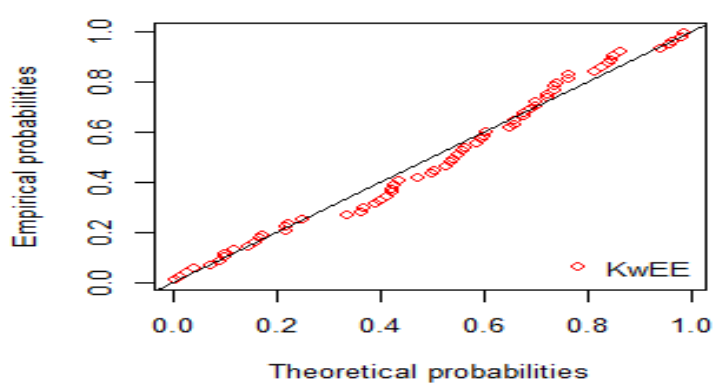

Figure 7: Histogram and fitted density plot, the plot of empirical and fitted cdf, P-P plot and Q-Q plot for the carbon data set in case of KwEE model.

\section{(ii) Data II}

The data represent fracture toughness of Alumina (Al2O3) (see Nadarajah and Kotz (2008)). We use the scaled-TTT plot to confirm the validity of the proposed model once more. The scaled-TTT plot is concave in Figure 8. It suggests that the hrf is increasing, confirming the accuracy of our model.

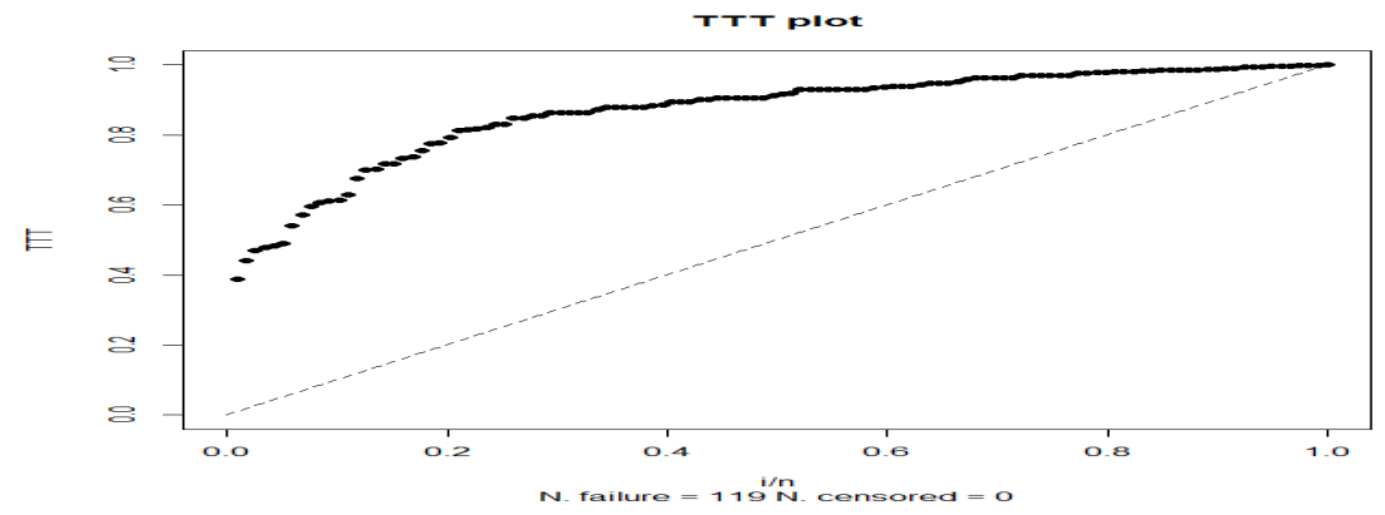

Figure 8: Scaled-TTT plot of the data II

The MLEs, AIC, BIC, $\mathrm{W}^{*}$, and $\mathrm{A}^{*}$ are listed in Table 7. The KwEE model, according to the results in Table 7, provides the best fit of all the models used here. The KwEE model holds lower the values of these numerical criteria. 
Table 7: The MLEs the proposed measures for second data set

\begin{tabular}{|ccccccc|}
\hline Model & \multicolumn{2}{c}{ MLEs } & AIC & BIC & $\boldsymbol{A}^{*}$ & $\boldsymbol{W}^{*}$ \\
\hline KwEE & $\hat{v}=6.025$ & $\hat{\delta}=23.170$ & $\mathbf{3 4 9 . 2 4 5}$ & $\mathbf{3 6 0 . 3 6 2}$ & $\mathbf{0 . 9 8 1}$ & $\mathbf{0 . 1 5 2}$ \\
& $\hat{\phi}=0.348$ & $\hat{\beta}=0.683$ & & & & \\
\hline gamma & $\hat{\phi}=15.523$ & $\hat{\lambda}=3.588$ & 358.736 & 364.295 & 2.342 & 0.388 \\
\hline GE & $\hat{\phi}=30.628$ & $\hat{\lambda}=0.910$ & 378.844 & 384.402 & 4.225 & 0.718 \\
\hline KwE & $\hat{\phi}=41.715$ & $\hat{\beta}=1.02$ & 380.842 & 386.400 & 5.100 & 0.966 \\
\hline L & $\hat{\theta}=0.397$ & & 536.795 & 539.575 & 25.114 & 4.993 \\
\hline GL & $\hat{\theta}=3.640$ & $\hat{\phi}=15.058$ & 360.543 & 368.880 & 2.326 & 0.386 \\
& $\hat{\beta}=7.983$ & & & & & \\
\hline EL & $\hat{\theta}=1.082$ & $\hat{\phi}=19.973$ & 375.315 & 380.873 & 3.877 & 0.657 \\
\hline
\end{tabular}

Figure 9 shows the histogram of the data set, the fitted pdf of the KwEE distribution, and the fitted pdfs of other comparable distributions for the second data set. The P-P plots and Q-Q plots for all models are also represented in Figure 9. Plots of empirical pdf, fitted cdf, as well as P-P and Q-Q plots for the KwEE model are represented in Figure 10. All the graphs show that the KwEE model yields a better fit in comparison to the competitor models. The results in Table 7 are similarly supported by these charts.

Histogram and theoretical densities

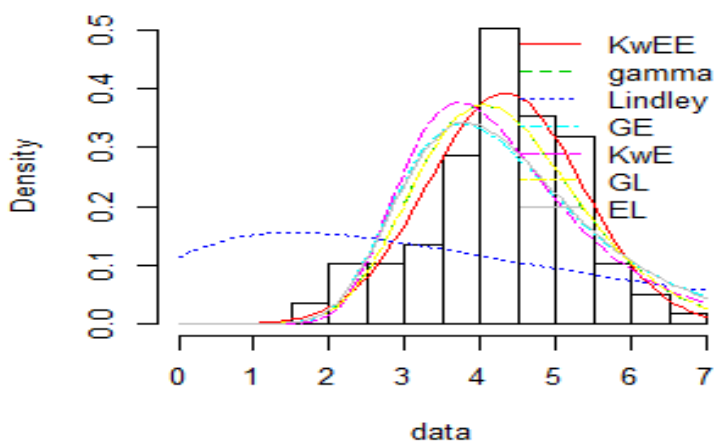

Empirical and theoretical CDFs

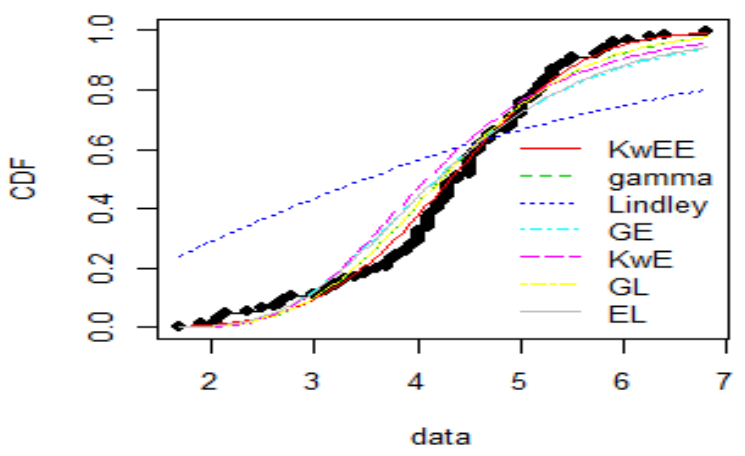

Q-Q plot

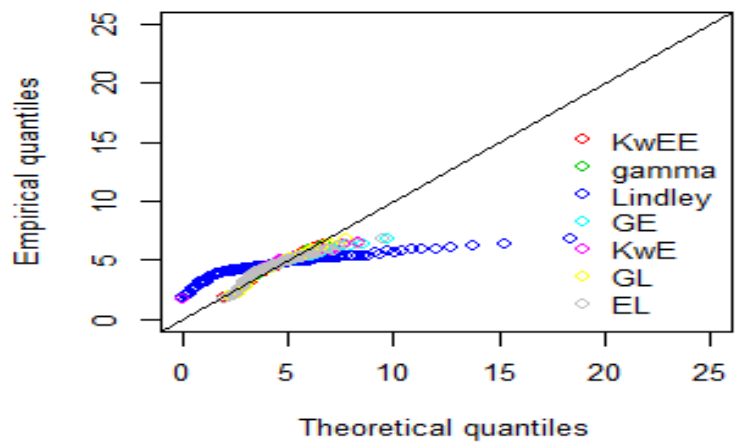

P-P plot

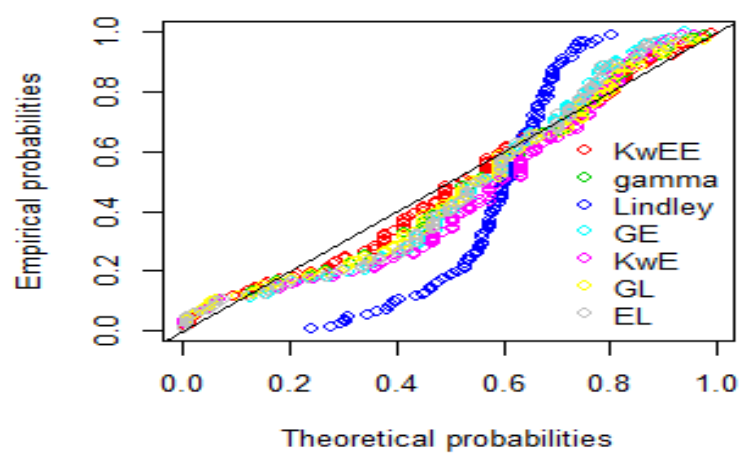

Figure 9: Histogram and fitted density plots, the plots of empirical and fitted cdfs, P-P plots and Q-Q plots for data II 
Histogram and theoretical densities

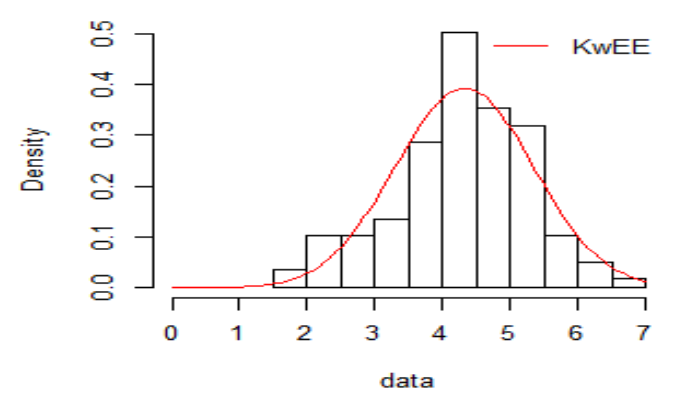

Empirical and theoretical CDFs

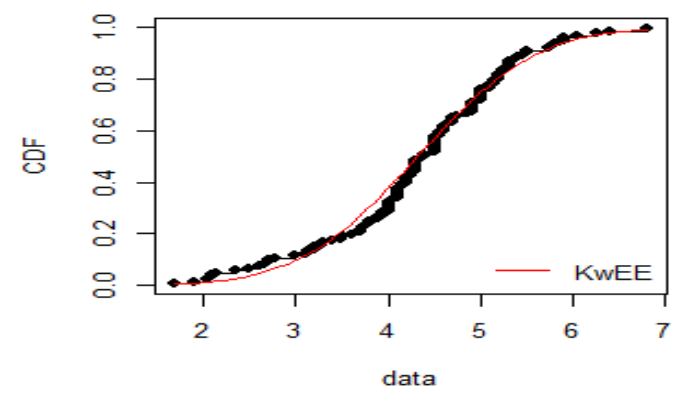

Q-Q plot
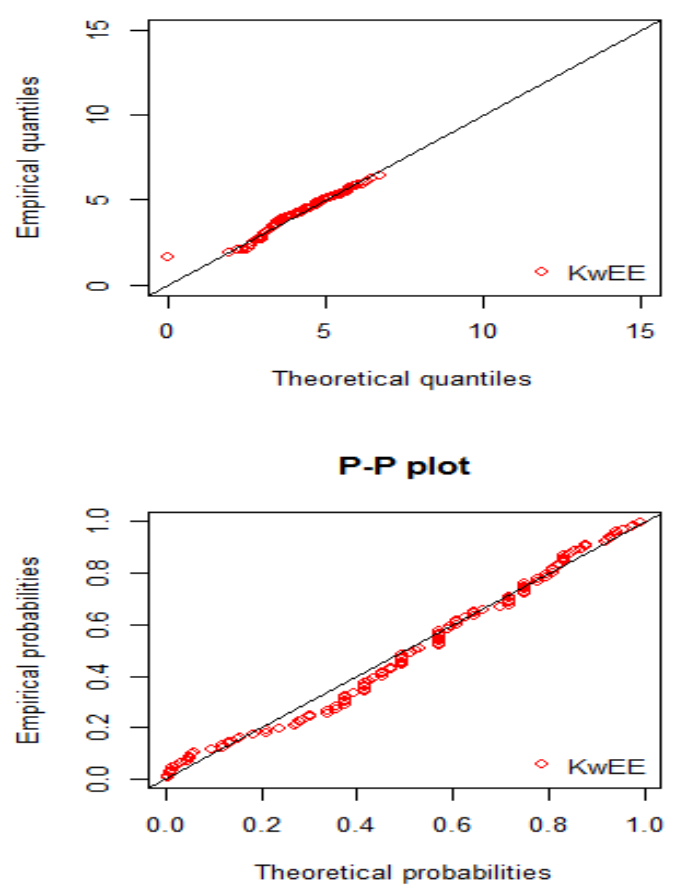

Figure 10: Histogram and fitted density plot, the plot of empirical and fitted cdf, P-P plot and Q-Q plot for the carbon data set in case KwEE model

\section{Summary and Conclusion}

Significant progress has been achieved in the generalization of some well-known lifetime models, which have been effectively applied to challenges in a variety of fields. In this paper, we introduce a four-parameter distribution obtained by applying the Kumaraswamy generator to the extended exponential distribution. A new generalization of the EE distribution called Kumaraswamy extended exponential distribution. The exponential, Kumaraswamy exponential, Kumaraswamy Lindley, Lindley, extended exponential, exponentiated Lindley, gamma, and generalized exponential distributions are among the specialized sub-models. Structure behavior of the new model is studied based on its some useful properties. The estimation of the model parameters is approached by maximum likelihood method. Simulation illustration is conducted in order to evaluate the performance of estimates for different sample sizes. Two applications of the KwEE model to the real data show that the new distribution can be used quite effectively to provide better fits then we expect that the proposed model may be an interesting alternative model for a wider range of statistical research.

\section{Acknowledgement}

The authors are deeply grateful to the anonymous referees and Editor for their insightful comments and recommendations, which resulted in this revised version.

\section{References}

1. Aarset, M. V. (1987). How to identify a bathtub hazard rate. IEEE Transactions on Reliability, 36(1), 106108.

2. Afify, A. Z. and Mead, M. E. (2017). On five-parameter Burr XII distribution: properties and applications. South African Statistical Journal, 51(1), 67-80.

3. Afify, A. Z. and Mohamed, O. A. (2020). A new three-parameter exponential distribution with variable shapes for the hazard rate: Estimation and applications. Mathematics, 8(1), 135. doi:10.3390/math8010135

4. Afify, A. Z., Zayed, M. and Ahsanullah, M. (2018). The extended exponential distribution and its applications. Journal of Statistical Theory and Applications, 17(2), 213-229. 
5. Barreto-Souza, W., Santos, A. H. S. and Cordeiro, G. M. (2010). The beta generalized exponential distribution. Journal of Statistical Computation and Simulation, 80(2), 159-172.

6. Çakmakyapan, S. and Kadilar, G. Ö. (2014). A new customer lifetime duration distribution: the Kumaraswamy Lindley distribution. International Journal of Trade, Economics and Finance, 5(5), 441444.

7. Cordeiro, G. M. and de Castro, M. (2011). A new family of generalized distributions. Journal of Statistical Computation and Simulation, 81(7), 883-898.

8. Cordeiro, G. M. and Lemonte, A. J. (2011). The $\beta$-Birnbaum-Saunders distribution: An improved distribution for fatigue life modeling. Computational Statistics \& Data Analysis, 55(3), 1445-1461.

9. Cordeiro, G. M., Nadarajah, S. and Ortega, E. M. (2012). The Kumaraswamy Gumbel distribution. Statistical Methods \& Applications, 21(2), 139-168.

10. Corless, R. M., Gonnet, G. H., Hare, D. E. G., Jeffrey, D. J. and Knuth, D. E. (1996). On the Lambert W function. Advances in Computational Mathematics, 5(1), 329-359.

11. de Andrade, T. A. N., Bourguignon, M. and Cordeiro, G. M. (2016). The exponentiated generalized extended exponential distribution. Journal of Data Science, 14(3), 393-413.

12. Elbatal, I., Louzada, F. and Granzotto, D. C. T. (2018). A new lifetime model: The Kumaraswamy extension exponential distribution. Biostatistics and Bioinformatics, 2, 1-9.

13. Gomes, A. E., da-Silva, C. Q., Cordeiro, G. M. and Ortega, E. M. (2014). A new lifetime model: the Kumaraswamy generalized Rayleigh distribution. Journal of statistical computation and simulation, 84(2), 290-309.

14. Gómez, Y., Bolfarine, H. and Gómez, H. W. (2014). A new extension of the exponential distribution. Revista Colombiana de Estad'istica, 37, 25-34.

15. Gupta, R. D. and Kundu, D. (1999). Generalized exponential distributions. Australian \& New Zealand Journal of Statistics, 41(2), 173-188.

16. Gupta, R. D. and Kundu, D. (2001). Exponentiated exponential family: an alternative to gamma and Weibull distributions. Biometrical Journal: Journal of Mathematical Methods in Biosciences, 43(1), 117130.

17. Hassan, A. S., Almetwally, E. M. and Ibrahim, G. M. (2021). Kumaraswamy inverted Topp-Leone distribution with applications to COVID-19 data. CMC-Computers, Materials \& Continua, 68(1), 337-358.

18. Hassan, A. S., Mohamd, R. E., Elgarhy, M. and Fayomi, A. (2018). Alpha power transformed extended exponential distribution: properties and applications. Journal of Nonlinear Sciences and Applications, $12(4), 62-67$.

19. Jodrá, P. (2010). Computer generation of random variables with Lindley or Poisson-Lindley distribution via the Lambert W function. Mathematics and Computers in Simulation, 81(4), 851-859.

20. Merovci, F. (2013). Transmuted exponentiated exponential distribution. Mathematical Sciences and Applications E-Notes, 1(2), 112-122.

21. Nadarajah, S. and Haghighi, F. (2011). An extension of the exponential distribution. Statistics, 45(6), 543558.

22. Nadarajah, S. and Kotz, S. (2008). Strength modeling using Weibull distributions. Journal of mechanical science and technology, 22(7), 1247-1254.

23. Nadarajah, S., Bakouch, H. S. and Tahmasbi, R. (2011). A generalized Lindley distribution. Sankhya B, 73(2), 331-359.

24. Nassar, M., Kumar, D., Dey, S., Cordeiro, G. M. and Afify, A. Z. (2019). The Marshall-Olkin alpha power family of distributions with applications. Journal of Computational and Applied Mathematics, 351, 41-53.

25. Rasekhi, M., Alizadeh, M., Altun, E., Hamedani, G., Afify, A. Z. and Ahmad, M. (2017). The modified exponential distribution with applications. Pakistan Journal of Statistics, 33(5), 383-398.

26. Ristić, M. M. and Balakrishnan, N. (2012). The gamma-exponentiated exponential distribution. Journal of Statistical Computation and Simulation, 82(8), 1191-1206.

27. Shahbaz, M. Q., Shahbaz, S. and Butt, N. S. (2012). The kumaraswamy-inverse Weibull distribution. Pakistan Journal of Statistics and Operation Research, 8(3), 479-489.

28. Tahir, M. H., Cordeiro, G. M., Mansoor, M., Zubair, M. and Alzaatreh, A. (2021). The Kumaraswamy Pareto IV distribution. Austrian Journal of Statistics, 50(5), 1-22.

29. Zakerzadeh, H. and Dolati, A. (2009). Generalized Lindley distribution. Journal of Mathematical Extension, 3, 13-25. 